Tropical Journal of Pharmaceutical Research March 2019; 18 (3): 485-490

ISSN: 1596-5996 (print); 1596-9827 (electronic)

(C) Pharmacotherapy Group, Faculty of Pharmacy, University of Benin, Benin City, 300001 Nigeria.

\title{
Effects of Xinwei granule on expression levels of cyclin D1 and its upstream genes in gastric intraepithelial neoplasia tissues
}

\author{
Jingbin Wang ${ }^{1,2}$, Cunguo $\mathrm{Yu}^{3,4 *}$, Xinyao $\mathrm{Liu}^{2}$, Yang Zhang ${ }^{1}$, Guoying Liang ${ }^{1}$, \\ Zhaoxia Liu ${ }^{1}$ \\ ${ }^{1}$ Department of Spleen and Stomach Disease, Heilongjiang University of Chinese Medicine, Haerbin, Heilongjiang Provence \\ 150040, ${ }^{2}$ Department of Spleen and Stomach Disease, Guangzhou University of Chinese Medicine-Shenzhen Hospital, \\ Shenzhen, Guangdong Province 518000, ${ }^{3}$ College of Electrical Engineering, Yanshan University, Qinhuangdao, Hebei \\ Province 066004, China, ${ }^{4}$ Department of Chinese Medicine, Qinhuangdao Haigang Hospital, Qinhuangdao, Hebei Province \\ 066000, China
}

*For correspondence: Email: yucunguocgc@163.com; Tel: 0086-335-3267453

\begin{abstract}
Purpose: To explore the effects of Xinwei granule (XWG) on low-grade gastric intraepithelial neoplasia (LGIN) and the underlying mechanisms.

Methods: To establish LGIN model, Wistar rats were treated with N-methyl-N'-nitrosoguanidine for 3 months. LGIN model rats were randomly grouped into five groups $(n=15)$, viz, negative control (NC), normal saline (NS) group, Xinwei granule (XWG) group, Weifuchun tablet (WFCT) group, and vatacoenayme tablet (VT) group. Normal rats $(n=17)$ served as negative control. Histological evaluation of gastric mucosa was undertaken using hematoxylin and eosin staining. Quantitative realtime polymerase chain reaction ( $q R T-P C R$ ), western blot, and immunohistochemical assays were performed to determine mRNA expressions, protein expression, and the distribution of cyclin D1, kruppel-like factor 4 (KLF4), and p21-WAF1-CIP1, respectively.

Results: Compared with LGIN group, the body weight of the rats increased in XWG, WFCT, and VT groups. The pathological characteristics of LGIN group were alleviated by XWG, WFCT and VT treatments. The positive expression of cyclin D1 was enhanced in LGIN group, but reduced in XWG, WFCT and VT groups. The expression levels of KLF4 and p21-WAF1-CIP1, upstream regulators of cyclin D1 reduced in LGIN groups. However, administration of XWG, WFCT and VT strengthened the expressions of KLF4 and p21-WAF1-CIP1. More importantly, the protective effects of XWG against LGIN were superior to those of WFCT and VT.

Conclusion: Xinwei granules alleviate LGIN in vivo by inhibiting cyclin D1 expression and enhancing KLF4 and p21-WAF1-CIP1 expression.
\end{abstract}

Keywords: Xinwei Granule, Cyclin D1, Kruppel-like factor 4, p21-WAF1-CIP1, Low-grade gastric intraepithelial neoplasia

\begin{abstract}
This is an Open Access article that uses a funding model which does not charge readers or their institutions for access and distributed under the terms of the Creative Commons Attribution License (http://creativecommons.org/licenses/by/4.0) and the Budapest Open Access Initiative (http://www.budapestopenaccessinitiative.org/read), which permit unrestricted use, distribution, and reproduction in any medium, provided the original work is properly credited.
\end{abstract}

Tropical Journal of Pharmaceutical Research is indexed by Science Citation Index (SciSearch), Scopus, International Pharmaceutical Abstract, Chemical Abstracts, Embase, Index Copernicus, EBSCO, African Index Medicus, JournalSeek, Journal Citation Reports/Science Edition, Directory of Open Access Journals (DOAJ), African Journal Online, Bioline International, Open-J-Gate and Pharmacy Abstracts 


\section{INTRODUCTION}

Gastric intraepithelial neoplasia (GIN) is a precancerous lesion of gastric cancer. The 5year survival rate in patients with gastric cancer at the early stage is closely associated with GIN treatment [1]. Gastric intraepithelial neoplasia can be divided into low-grade gastric intraepithelial neoplasia (LGIN) and high-grade gastric intraepithelial neoplasia (HGIN) [2]. Highgrade gastric intraepithelial neoplasia is considered to be suitable for endoscopic or surgical local resection [3]. However, the strategies for the effective diagnosis and therapy of LGIN still need further development. Uncontrolled cell cycle progression can lead to carcinogenesis and the growth of human cancers $[4,5]$. Cell cycle progression is modulated by cyclin-dependent kinases [6]. Cyclin D1 is an important cell cycle regulator, which plays a role in human cancer progression [7-9]. The involvement of cyclin D1 in gastric cancer [10] has been described previously. Moreover, Kruppel-like factor 4 (KLF4), a tumor regulator can modulate the expression of cyclin D1 [11]. p21-WAF1-CIP1 is a cyclin-dependent kinase inhibitor that inhibits the cyclin complex [12]. In addition, disruption of KLF4 [13] and p21-WAF1CIP1 [14] expression has been observed in gastric cancer. Therefore, investigating the expression of cyclin D1, KLF4, and p21-WAF1CIP1 may be beneficial for understanding the mechanisms of LGIN development.

In this study, the effects of Xinwei granule, weifuchun tablets, and Vatacoenayme tablets on LGIN, and investigated the mechanism underlying the effects caused by these drugs was investigated.

\section{EXPERIMENTAL}

\section{Animal grouping}

Seventy-seven male Wistar rats (body weight, 180 - $200 \mathrm{~g} ; 6$ - 8 weeks) were purchased from the Animal Experiment Center of the Shanghai Chinese Academy of Sciences. The animals were kept at $21-22{ }^{\circ} \mathrm{C}$, with a $12 \mathrm{~h} / 12 \mathrm{~h}$ light/dark cycle and $50-60 \%$ humidity. To establish the LGIN model, N-methyl-N-nitro-N'nitrosoguanidine (MNNG; $25 \mu \mathrm{g} / \mathrm{mL}$; Aldrich Chemical Co. Ltd, Milwaukee, WI) was administered to 60 rats in animals' drinking water (placed in dark bottles) for 3 months. Subsequently, the rats were randomly placed in four groups ( $n=15$ per group) as follows: Rat in the negative saline (NS) group were treated with saline, by gastrogavage; the Xinwei Granule (XWG) group were administered with XWG (4 $\mathrm{g} / \mathrm{kg} /$ day) by gastrogavage; rats in the Weifuchun Tablets (WFCT) group were administered with WFCT $(0.72 \mathrm{~g} / \mathrm{kg} /$ day $)$ by gastrogavage; rats in the Vatacoenayme Tablets (VT) group were administered with VT (1 $\mathrm{g} / \mathrm{kg} /$ day $)$ by gastrogavage.

Drug treatment lasted for 8 weeks. Normal rats ( $n=17$ ) without treatment served as the negative control (NC) group. No animals died during the experiments before they were sacrificed. Anesthesia was provided via intraperitoneal injection of $10 \%$ chloral hydrate $(0.3 \mathrm{~mL} / 100 \mathrm{~g}$ body weight). Subsequently, the animals were sacrificed by tail vein air embolization. An approximately $0.5 \mathrm{~mm} \times 2.0 \mathrm{~mm}$ section of tissue was excised from the gastric antrum and fixed in $4 \%$ paraformaldehyde. All animal experiments were performed according to the Institutional Animal Care and Use Committee (IACUC) [15] and approved by the Ethics Committee of Heilongjiang University of Chinese Medicine (approval no. HU17051682).

\section{Haematoxylin and eosin (H \& E) staining and immunohistochemical (IHC) assay}

The tissues were embedded in paraffin and were cut into $4-\mu \mathrm{m}$ slices. The slides were dewaxed using xylene. After dehydrating with gradient ethanol, the slides were stained with hematoxylin for $15 \mathrm{~min}, 1 \%$ hydrochloric acid alcohol for $15 \mathrm{~s}$, and $1 \%$ eosin staining for $1 \mathrm{~min}$. The extent of LGIN was classified as none, moderate, and severe, according to the degree of gastromucosal dysplasia [16]. The slides were evaluated by a pathologist in a blinded manner.

For IHC assay, slides were boiled in sodium citrate buffer $(\mathrm{pH} 6.0,10 \mathrm{mM})$ for antigen retrieval. The sections were allowed to cool to room temperature and incubated in hydrogen peroxide $(3 \%)$ for $10 \mathrm{~min}$. The slides were blocked by incubating with $10 \%$ normal goat serum (Thermo Fisher Scientific) at $37^{\circ} \mathrm{C}$. After $30 \mathrm{~min}$, slides were incubated with anti-cyclin D1 (cat. no. ab226977; dilution, 1:300, Abcam, Cambridge, UK), anti-KLF4 (cat. no. ab106629; dilution, 1:500, Abcam), and anti-p21-WAF1CIP1 (cat. no. ab80633; dilution, 1:200, Abcam) overnight at $4{ }^{\circ} \mathrm{C}$. Secondary biotin-labeled antibodies were added to the sections and incubated for $1 \mathrm{~h}$ at room temperature. Next, the slides were incubated with horseradish peroxidase-linked streptavidin at room temperature for $30 \mathrm{~min}$. Finally, the slides were incubated with diaminobenzidine (DAB) for $5 \mathrm{~min}$ at room temperature. Images were observed under a light microscope at magnification of $\times 100)$. 


\section{Western blotting}

Tissues proteins were isolated using Tissue or Cell Total Protein Extraction Kit (Sangon, Shanghai, China). The denatured proteins were separated using $8 \%$ sodium dodecyl sulfatepolyacrylamide gel electrophoresis (SDS-PAGE). After transfer onto a polyvinylidene fluoride membrane (Millipore, Bedford, MA), the proteins were blocked using $5 \%$ non-fat milk for 2 hours at room temperature. Next, the membrane was incubated with primary antibodies, including anticyclin D1 (cat. no. ab40754; dilution, 1:1000), anti-KLF4 (cat. no. PA5-23184; dilution, 1:500, Thermo Fisher Scientific), anti-p21-WAF1-CIP1 (cat. no. ab80633; dilution, 1:200, Abcam), and anti-H3 (ab1791, 1:2000, Abcam), at $4^{\circ} \mathrm{C}$ overnight. The membrane was then incubated with HRP-linked secondary antibodies, goat antirabbit IgG (cat. no. ab6721; 1:5000) and goat anti-mouse IgG (cat. no. ab6785; 1:5000, Abcam), at room temperature for $1.5 \mathrm{~h}$. The protein blots were visualized using enhanced chemiluminescence (Pierce Biotechnology, IL, USA).

\section{Quantitative real-time polymerase chain reaction (qRT-PCR)}

Total RNA from tissues was isolated using the TRIzol reagent (Invitrogen, Carlsbad, CA, USA). Complementary DNA (cDNA) synthesis was performed using a first-strand cDNA kit (Takara, Tokoyo, China) according to the manufacturer's protocols. The SYBR Green PCR kit (Thermo) was used for PCR amplification on an ABI7300 Thermocycler (Applied Biosystems, Foster City, CA, USA). The primer sequences used are listed in Table 1. Relative mRNA expression levels were analyzed based on $2^{-\triangle \Delta C T}$ calculation.

\section{Statistical analysis}

GraphPad Prism 6.0 (GraphPad Software, Inc, La Jolla, CA, USA) was used to perform data analysis. Differences among groups were analyzed by one-way ANOVA followed by Tukey's test. Data are expressed as mean \pm standard deviation (SD). $P<0.05$ was considered statistically significant.

\section{RESULTS}

\section{Xinwei granule reduced the pathological changes in LGIN rats}

To investigate the efficiency of Xinwei granule on LGIN, the effect of Xinwei Granule on the morphology of the LGIN rats was first observed.
The animals in NC group showed quick reaction and granular stools. The LGIN model rats appeared to exhibit depressed mental state, hair shedding, reduced hair glossiness, decreased activity, and loss of appetite, but these symptoms were relieved in the XWG, WFCT, and VT groups. Furthermore, as shown in Figure 1, the body weight of LGIN model rats was reduced when compared with that of the negative control (NC) group (378.4 \pm 14.0 vs. $530.2 \pm 12.3 \mathrm{~g}, p<$ $0.001)$. However, the body weight loss of the LGIN rats was protected by the administration of XWG $(478.4 \pm 14.0 \mathrm{~g}$ vs. $378.4 \pm 14.0 \mathrm{~g}, p<$ $0.001)$, WFCT $(458.4 \pm 14.0 \mathrm{~g}$ vs. $378.4 \pm 14.0 \mathrm{~g}$, $p<0.001)$, or VT $(450.4 \pm 14.0$ g vs. $378.4 \pm$ $14.0 \mathrm{~g}, p<0.001)$, unlike the NS group. The pathological changes of rats were more pronounced in the XWG group when compared with those in the WFCT and VT groups $(p<$ 0.05).

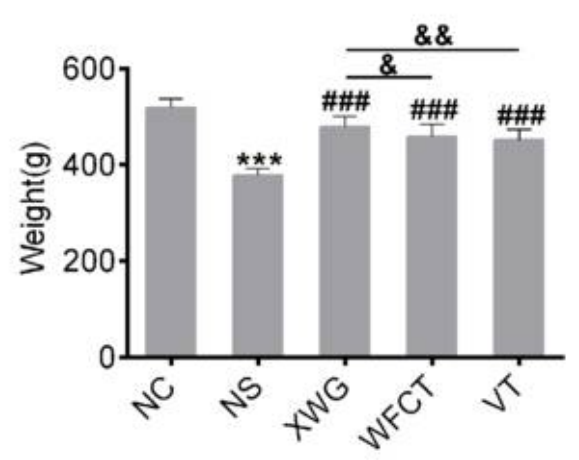

Figure 1: Body weight of animals in the negative control (NC) group, Xinwei Granule (XWG) group, Weifuchun Tablet (WFCT) group, and Vatacoenayme Tablet (VT) group. ${ }^{\star * *} P<0.001$ vs. NC group; ${ }^{\# \# ~} p<$ 0.001 vs. NS group; ${ }^{\&} p<0.05 ;{ }^{\& \&} p<0.01$ vs. XWG group

Xinwei granule reversed changes in the
morphology of gastric mucosa in LGIN rats

First, the effect of Xinwei granule on the morphology of gastric mucosa was studied using H\&E staining. The results (Figure 2 A) showed that rats in the NC group did not display LGIN symptoms. All the cases in the NS group exhibited severe LGIN. In contrast, there were only two cases in the XWG group, four cases in the WFCT group, and four cases in the VT group, indicating that the degree of LGIN was mitigated by XWG, WFCT, and VT. In addition, the gastric mucosa folds were regular and well stretched in the NC group, but the NS group showed reduced gastric mucosa folds and increased fibroplasia. In contrast to the NS group, treatment with XWG, WFCT, or VT relieved the pathological changes in LGIN 
(Figure $2 \mathrm{~B}$ ). These results indicated that Xinwei Granule mitigated the effects of LGIN.

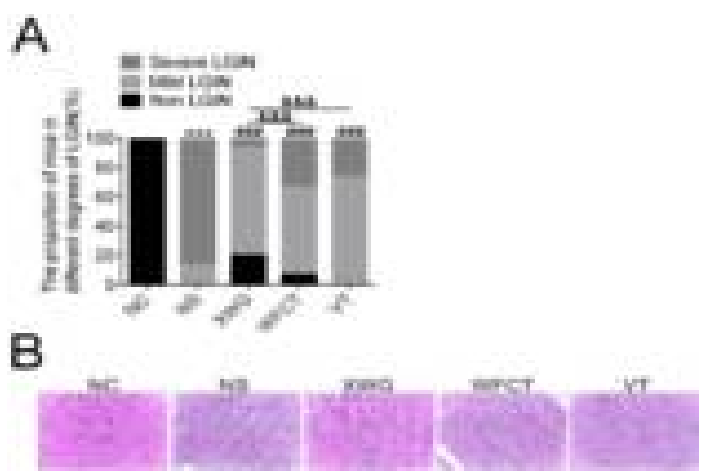

Figure 2: (A) Cases of LGIN in the negative control (NC), XWG, WFCT, and VT groups were analyzed; ${ }^{* *} p$ $<0.001$ vs. the NC group; ${ }^{\# \# \#} p<0.001$ vs. the NS group; $\& \& \& p 0.001$ vs. the XWG group. (B) Histological changes in the NC, XWG, WFCT, and VT groups were detected using $\mathrm{H}$ \& $\mathrm{E}$ staining

\section{Effect of Xinwei granule on the distribution of cyclin D1, KLF4, and p21WAF1/CIP1 in LGIN tissues}

To further illustrate the molecular mechanism underlying the effects of Xinwei Granule, the expression of cyclin D1, KLF4, and p21WAF1/CIP1 was examined. Immunohistochemistry assays (Figure 3) revealed that cyclin D1 staining was stronger in the NS group than in the NC group, whereas cyclin D1 staining was weaker in the XWG, WFCT, and VT groups than in the NS group. Moreover, staining for KLF4 and p21WAF1/CIP1 was lower in the NS group, but was enhanced by XWG, WFCT, and VT treatment. Among the three drug treatment groups, the effect on cyclin D1, KLF4 and p21WAF1/CIP1 staining appeared to be most remarkable in the XWG group. These results suggest that Xinwei granule decreased the expression distribution of cyclin D1 and increased the expression distribution of KLF4 and p21WAF1/CIP1 in LGIN tissues.

\section{Xinwei granule regulated protein and mRNA expression levels of cyclin D1, KLF4, and p21WAF1/CIP1 in LGIN tissues}

The protein and mRNA expression levels of cyclin D1, KLF4, and p21WAF1/CIP1 were measured. The results (Figure $4 \mathrm{~A}$ ) showed that the increased cyclin D1 protein expression in LGIN rats was reduced in the XWG $(p<0.001)$ and VT $(p<0.001)$ groups. The reduced protein expression of KLF4 in LGIN group was protected in the XWG $(p<0.001)$, WFCT $(p<0.01)$, and VT $(p<0.01)$ groups. Similarly, protein expression of p21WAF1/CIP1 was recovered in the XWG $(p<0.001)$, WFCT $(p<0.05)$, and VT $(p<0.05)$ groups when compared with the NS group.

Importantly, the effect on cyclin D1, KLF4, and p21WAF1/CIP1 expressions in the XWG group were more significant than those in the WFCT ( $p$ $<0.05)$ and VT $(p<0.05)$ groups. The mRNA expression profiles of cyclin D1, KLF4, and p21WAF1/CIP1 in this study matched their respective protein profiles (Figure $4 \mathrm{~B}$ ). These results suggest that the expression of cyclin D1, KLF4 and p21WAF1/CIP1 may be modulated by Xinwei Granule at both the translational and transcriptional levels.

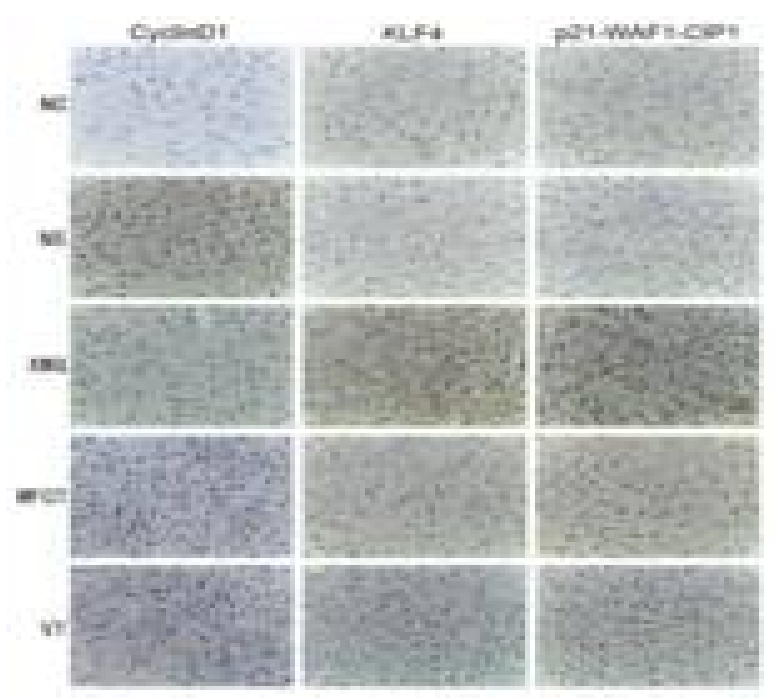

Figure 3: Expression patterns of cyclin D1, KLF4, and p21WAF1/CIP1 in the negative control (NC), XWG, WFCT, and VT groups were detected using IHC staining

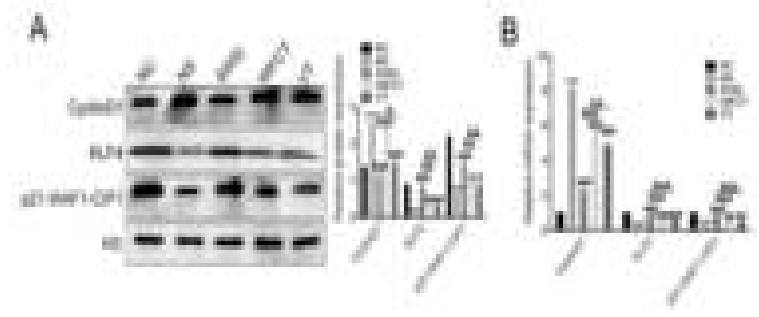

Figure 4: (A) Protein expression of cyclin D1, KLF4 and p21WAF1/CIP1 in the negative control (NC), XWG, WFCT, and Vatacoenayme Tablets VT groups were assayed using western blot; $p<0.01$ and ${ }^{* * *} p<$ 0.01 vs. NC group; ${ }^{\# \#} p<0.001$ vs. NS group; ${ }^{\&} p<$ $0.05,{ }^{\& \&} p<0.01$, and ${ }^{\& \&} p<0.001$ vs. XWG group. (B) mRNA expression levels of cyclin D1, KLF4, and p21WAF1/CIP1 in the negative control (NC), XWG, WFCT, and VT groups were assayed using gPCR; ${ }^{* * *} p<0.01$ vs. the NC group; ${ }^{\# \#} p<0.01$, and ${ }^{\# \# \#} p<$ 0.001 vs. NS group; ${ }^{\&} p<0.01$ and ${ }^{\& \& \&} p<0.001$ vs. XWG group 


\section{DISCUSSION}

It has been reported that patients with intermediate stages of gastric cancer often manifest symptoms like chronic gastritis and intestinal metaplasia [17]. The 5-year survival rate of patients with early-stage gastric cancer is significantly higher than that of advanced-stage patients [18]. It has been reported that LGIN can often be reversed, and only a few of these cases developed into invasive cancer [19]. Therefore, effective treatment and early detection of LGIN is significant in preventing the occurrence of gastric cancer.

In this study, it was found that administration of Xinwei granule, Weifuchun Tablets, and Vatacoenayme Tablets relieved LGIN to varying degrees. Reduced body weight in LGIN rats was protected by treatment with these three agents. The activity, and diet of the LGIN rats were improved by drug treatment and Xinwei Granule was more effective than the other two drugs. These results indicate that the administration of Xinwei Granule may improve the quality of life in patients with LGIN.

Histologically, little chronic inflammation was observed in the NC group, whereas hyperplasia was most obvious in the LGIN model group. In contrast, fibroplasia in LGIN tissues was blocked and the number of severe LGIN cases was reduced in the XWG, WFCT, and VT groups. The alleviation of LGIN appeared greater after treatment with Xinwei Granule than with Weifuchun Tablets or Vatacoenayme Tablets. Taken together, these results showed that Xinwei Granule effectively mitigated the symptoms of LGIN and prevented carcinogenesis in gastric mucosa.

It is believed that cellular proliferation is a tightly regulated and ordered modulated progress, which is fundamental for cellular homeostasis [20]. Cyclin D1 serves as a cell cycle regulatory switch in living cells [21]. Thus, examining cyclin D1 levels in LGIN may be useful for the early diagnosis of LGIN and for the understanding of the molecular mechanisms underlying the effects of Xinwei Granule on LGIN. Because KLF4 [17] and p21-WAF1-CIP1 [22] are upstream regulators of cyclin D1, this study analyzed the expression levels ofKLF4, p21-WAF1-CIP1, as well as cyclin D1 in LGIN rats. The present results showed that cyclin D1 expression was increased in LGIN tissues, but was decreased in the XWG, WFCT and VT groups.

Furthermore, the effects of Xinwei Granule were more pronounced than those of Weifuchun
Tablets and Vatacoenayme Tablets. Cell cycle progression occurs when the intracellular level of cyclin D1 is elevated [21]. Therefore, the present results suggested that increased cyclin D1 expression in LGIN tissues facilitated malignant growth of gastric tissues. Interestingly, drug treatment resulted in reduced expression of cyclin D1 in LGIN tissues, which suggests that drug treatment was capable of preventing carcinogenesis in the gastric mucosa. Moreover, the expression levels of KLF4 and p21-WAF1CIP1 were upregulated in the drug treatment groups compared with those of the LGIN model group, suggesting that the activation of KLF4 and p21-WAF1-CIP1 may suppress LGIN.

It has been reported that KLF4 [17] and p21WAF1-CIP1 [23] can serve as tumor suppressors; this is consistent with the current results of this study. Nevertheless, KLF4 and p21-WAF1-CIP1 may also promote tumor occurrence due to their dual role in cancer progression $[24,25]$. The present results suggest that measuring the expression of cyclin D1 and its regulators may be useful predictors of LGIN. In summary, it can be concluded that treatment with Xinwei Granule inhibited cyclin D1 expression and increased KLF4 and p21-WAF1CIP1 expression, which may reduce uncontrolled cell cycle progression in LGIN tissues, and thereby prevent gastric carcinogenesis.

\section{CONCLUSION}

The findings of this work indicate that Xinwei granule alleviates LGIN and regulates the expression of cyclin D1 and its upstream genes in LGIN tissues to a greater extent than weifuchun tablets and vatacoenayme tablets. Thus, XWG may prove to be an effective agent for the clinical treatment of LGIN and may open up potential detectable targets for the diagnosis of LGIN. However, one limitation is that the definite pharmacological effects of the drugs, especially that of Xinwei granule, were not validated in the current study, and this is worthy of further in-depth investigation.

\section{DECLARATIONS}

\section{Acknowledgement}

This research was supported as part of a project funded by "Natural Science Foundation of Heilongjiang Province of China" (Grant no. H2017071) , Project of Administration of Traditional Chinese Medicine of Hebei Province of China (Grant no.2018385). 


\section{Conflict of interest}

The authors declare that no conflict of interest is associated with this work.

\section{Contribution of authors}

We declare that this work was done by the authors named in this article and all liabilities pertaining to claims relating to the content of this article will be borne by the authors. D Jingbin Wang and Cunguo $\mathrm{Yu}$ designed all the experiments and revised the paper. Yang Zhang, Guoying Liang and Zhaoxia Liu performed the experiments, and Xinyao Liu wrote the paper.

\section{REFERENCES}

1. Zhang HP, Yang $S$, Chen WH, Hu TT, Lin J. The diagnostic value of confocal laser endomicroscopy for gastric cancer and precancerous lesions among Asian population: a system review and meta-analysis. Scand $J$ Gastroenterol. 2017; 52(4): 382-388.

2. Ling $Y Z$, Dai J, Yun JZ, Han BX, Zhi ZG, Xiao BL. Endoscopic resection for gastric epithelial neoplasia: How to solve pathological discrepancy and achieve curative resection? J Digest Dis. 2013; 14(5): 231-237.

3. Feng WX, Zhou YC, Gui J, Ning Y, Fang SJ. Differential gene expression profiling of gastric intraepithelial neoplasia and early-stage adenocarcinoma. World $\mathrm{J}$ Gastroenterol. 2014; 20(47): 17883-17893.

4. Arung E, Wicaksono B, Ayupriyanti Handoko Y, Kusuma I, Yulia D, Sandra F. Anti-Cancer Properties of Diethylether Extract of Wood from Sukun (Artocarpus altilis) in Human Breast Cancer (T47D) Cells, vol. 8; 2009.

5. Hua $X$, Wen YP. In vitro antitumour activity of Sesamum indicum Linn flower extracts. Trop J Pharm Res. 2010; 9(5): 455-462.

6. Malumbres M, Barbacid M. Cell cycle, CDKs and cancer: a changing paradigm. Nature Rev Cancer. 2009; 9(3): 153-166.

7. Jiang $W$, Zhang YJ, Kahn SM, Hollstein MC, Santella RM, Lu SH, Harris CC, Montesano R, Weinstein IB. Altered expression of the cyclin $D 1$ and retinoblastoma genes in human esophageal cancer. P Natl Acad Sci USA. 1993; 90(19): 9026-9030.

8. Lamb J, Ramaswamy S, Ford HL, Contreras B, Martinez RV, Kittrell FS, Zahnow CA, Patterson N, Golub TR, Ewen ME. A Mechanism of Cyclin D1 Action Encoded in the Patterns of Gene Expression in Human Cancer. Cell. 2003; 114(3): 323-334.

9. Kim JK, Diehl JA. Nuclear cyclin D1: An oncogenic driver in human cancer. J Cell Physiol. 2010; 220(2): 292-296.

10. Chen $B$, Zhang $X Y$, Zhang $Y J$, Zhou $P$, Gu $Y$, Fan DM. Antisense to cyclin $D 1$ reverses the transformed phenotype of human gastric cancer cells. World $\mathrm{J}$ Gastroenterol. 1999; 5(1): 18-21.

11. Choi WJ, Youn SH, Back JH, Park S, Park EJ, Kim KJ, Park HR, Kim AL, Kim KH. The role of KLF4 in UVBinduced murine skin tumor development and its correlation with cyclin D1, p53, and p21(Waf1/Cip1) in epithelial tumors of the human skin. Arch Dermatol Res. 2011; 303(3): 191-200.

12. Xiong $Y$, Hannon $G J$, Zhang $H$, Casso $D$, Kobayashi $R$, Beach D. p21 is a universal inhibitor of cyclin kinases. Nature. 1993; 366: 701-712.

13. Cho YG, Song JH, Kim CJ, Nam SW, Yoo NJ, Lee JY, Park WS. Genetic and epigenetic analysis of the KLF4 gene in gastric cancer. Apmis. 2010; 115(7): 802-808.

14. Wei Z, Jiang $X$, Qiao $H$, Zhai B, Zhang L, Zhang $Q$, Wu $Y$, Jiang $H$, Sun $X$. STAT3 interacts with Skp2/p27/p21 pathway to regulate the motility and invasion of gastric cancer cells. Cellul Signal. 2013; 25(4): 931-938.

15. Moore FR. Institutional Animal Care and Use Committee (IACUC). Atla Abstracts. 2014; 13(13): 236-239.

16. Rogers $A B$, Taylor NS, Whary MT, Stefanich ED, Wang TC, Fox JG. Helicobacter pylori but not High Salt Induces Gastric Intraepithelial Neoplasia in B6129 Mice. Cancer Res. 2005; 65(23): 10709-10715.

17. Boussioutas A, Li H, Liu J, Waring P, Lade S, Holloway AJ, Taupin D, Gorringe K, Haviv I, Desmond PV. Distinctive Patterns of Gene Expression in Premalignant Gastric Mucosa and Gastric Cancer. Cancer Res. 2003; 63(10): 2569-2577.

18. Butte JM, Torres J, Viviani P, Duarte I, Crovari F, Guzmán S, Cabrera R, Pedemonte J, Llanos O. [Long term survival of patients operated for early gastric cancer]. Revista Médica De Chile. 2008; 136(11): 14241433.

19. Dixon MF. Gastrointestinal epithelial neoplasia: Vienna revisited. Gut. 2002; 51(1): 130-131.

20. Warwick GP. Effect of the cell cycle on carcinogenesis. Federation Proceed. 1971; 30(6): 1760-1769.

21. Stacey DW. Cyclin D1 serves as a cell cycle regulatory switch in actively proliferating cells. Curr Op Cell Bio. 2003; 15(2): 158-163.

22. Brennan DJ, Ek S, Doyle E, Drew T, Foley M, Flannelly G, O'Connor DP, Gallagher WM, Kilpinen S, Kallioniemi $O P$ et al. The transcription factor Sox11 is a prognostic factor for improved recurrence-free survival in epithelial ovarian cancer. Eur J Cancer. 2009; 45(8): 1510-1517.

23. Nandakumar V, Vaid M, Katiyar SK. (-)-Epigallocatechin3-gallate reactivates silenced tumor suppressor genes, Cip1/p21 and p16INK4a, by reducing DNA methylation and increasing histones acetylation in human skin cancer cells. Carcinogenesis. 2011; 32(4): 537-544.

24. Parveen A, Akash MS, Rehman K, Kyunn WW. Dual Role of p21 in the Progression of Cancer and Its Treatment. Crit Rev Euk Gene. 2016; 26(1): 49-57.

25. Yang WT, Zheng PS. Dual Roles of KLF4 as a Tumor Suppressor or Oncogene. Forum Immunopathol Dis Ther. 2016; 7: 1-2. 